\title{
Tradução para a língua portuguesa e validação da escala de Braden $Q$ para avaliar o risco de úlcera por pressão em crianças
}

\author{
Portuguese translation and validation of the Braden $Q$ scale for predicting pressure ulcer risk in pediatric patients
}

\author{
Ana Claudia A. R. Maia', Donata M. S. Pellegrino², Leila Blanes³, Gal Moreira Dini', Lydia Masako Ferreira ${ }^{5}$
}

RESUMO

Objetivo: Traduzir para a língua portuguesa, adaptar ao contexto cultural brasileiro e testar as propriedades de medidas, reprodutibilidade e validade da escala de Braden $Q$.

Métodos: A escala de Braden Q foi traduzida e adaptada de acordo com metodologia aceita internacionalmente. Realizouse tradução e tradução reversa do instrumento, intercaladas de revisões feitas por comitê multisciplinar. $\mathrm{Na}$ fase de adaptação cultural, três grupos de dez enfermeiras avaliaram a versão brasileira da escala de Braden Q até obter seu entendimento integral. Na validação da reprodutividade, outras duas enfermeiras aplicaram a versão brasileira em crianças internadas na UTI em tempos diferentes, sendo que a primeira enfermeira avaliou também em um segundo momento. Na análise estatística, para testar a consistência interna da escala, foi calculado o $\alpha$ de Crombach e, para testar a reprodutividade, o teste intraclasse e a correlação de Spearman.

Resultados: No processo de tradução e retrotradução, não houve diferença nas escalas feitas pelos diferentes tradutores. $\mathrm{Na}$ adaptação cultural realizada pelas 30 enfermeiras, todos os itens da escala foram considerados relevantes. A consistência interna testada pelo $\alpha$ de Crombach foi de 0,936; a correlação intraclasse da reprodutividade intraobservador foi de 0,995 e da reprodutividade interobservador foi de 0,998, ambas apontadas como excelentes.

Conclusões: A escala de Braden $Q$ foi traduzida e adaptada com sucesso, demonstrando ser válida e reprodutível.

Palavras-chave: úlcera por pressão; pediatria; unidades de terapia intensiva.

Instituição: Disciplina de Cirurgia Plástica da Escola Paulista de Medicina da Universidade Federal de São Paulo (EPM/Unifesp), São Paulo, SP, Brasil ${ }^{1}$ Mestre em Ciências pelo Programa de Pós-Graduação em Cirurgia Plástica da EPM/Unifesp, São Paulo, SP, Brasil

${ }^{2}$ Aluna do Programa de Pós-Graduação em Cirurgia Plástica da EPM/ Unifesp, São Paulo, SP, Brasil

${ }^{3}$ Doutora em Ciências pelo Programa de Pós-Graduação em Cirurgia Plástica da EPM/Unifesp, São Paulo, SP, Brasil

${ }^{4}$ Professor Afiliado da Disciplina de Cirurgia Plástica da EPM/Unifesp, São Paulo, SP, Brasil

${ }^{5}$ Professora Titular e Chefe da Disciplina de Cirurgia Plástica da EPM/ Unifesp, São Paulo, SP, Brasil

\section{ABSTRACT}

Objective: To translate and culturally adapt the Braden $\mathrm{Q}$ scale into a Portuguese version and to test its properties (reliability and validity).

Methods: The Braden Q scale was translated and adapted according to internationally accepted methodology. The instrument was forward and back translated, and the translations were reviewed by a multidisciplinary committee. In the cultural adaptation process, three groups of ten nurses each interpreted the Brazilian version of the Braden Q scale until they fully understood the instrument. In order to evaluate the reliability of the Brazilian version, two other nurses administered the tool to pediatric ICU patients at different time points; the first nurse administered the instrument also in a second time. Statistical analysis was performed using Cronbach's $\alpha$ to evaluate the internal consistency of the scale, and the Spearman and intra-class correlation coefficients were calculated as a measure of reliability.

Results: There were no differences between scales translated by different translators during the forward and back translation process. All items of the scale culturally adapted by the 30 nurses were considered relevant. Cronbach's $\alpha$ for internal consistency was 0.936 ; intra-class correlation coefficient for intra-rater reliability was 0.995 and for inter-rater reliability was 0.998 , both indicating high reliability.

Conclusions: The Braden Q scale was successfully translated and adapted, and demonstrated validity and reliability.

Key-words: pressure ulcer; pediatrics; intensive care units.

Endereço para correspondência:

Ana Claudia A. R. Maia

Rua Napoleão de Barros, 715, $4^{\circ}$ andar - Vila Clementino

CEP 04024-002 - São Paulo/SP

E-mail: ana-claudia-maia@uol.com.br

Conflito de interesse: nada a declarar

Recebido em: 7/9/2010

Aprovado em: 20/1/2011 


\section{Introdução}

A assistência adequada à criança hospitalizada pressupõe o reconhecimento de suas necessidades específicas pela equipe multidisciplinar, considerando-se as diferentes etapas de seu desenvolvimento ${ }^{(1,2)}$. Padrões de atendimento, indicativos da qualidade de assistência, têm sido estudados, beneficiando pacientes hospitalizados ${ }^{(3)}$. A integridade da pele participa na composição dos resultados positivos para o paciente ${ }^{(4)}$. Contraditoriamente, a manutenção da integridade da pele da criança, especialmente quando seu estado é crítico, nem sempre é considerada uma prioridade ${ }^{(5,6)}$. A úlcera por pressão (UP) localizada, geralmente sobre uma proeminência óssea, constitui-se em uma área de dano na pele ou em estruturas subjacentes, devido à pressão, fricção e/ou suas combinações ${ }^{(7,8)}$. Trata-se de tema importante na área da saúde por sua complexidade. Agravantes como a infecção, o desfiguramento e as sequelas aumentam o tempo de internação, o sofrimento e o custo do seu tratamento ${ }^{(1,6,9-13)}$.

Quanto mais imatura a criança e mais crítico seu estado de saúde, menos tolerantes a pele e as estruturas de suporte e maior o risco de desenvolver úlceras por pressão ${ }^{(14,15)}$. Estudos realizados na população pediátrica norte-americana revelam prevalência de UP de 0,47 a $17 \%^{(4,16-18)}$. Destaca-se sua ocorrência em doenças limitantes, em internações em unidades de terapia intensiva ou no pós-operatório ${ }^{(19)}$. Os fatores de risco mais frequentes, relacionados ao desenvolvimento de UP em crianças, são a imobilidade, a presença da força de fricção e o cisalhamento, a desnutrição, a perfusão tecidual e a oxigenação alteradas $^{(12-14)}$. A prevenção efetiva requer avaliação dos fatores de risco para essa população.

Instrumentos específicos, validados para detecção do risco de a criança desenvolver UP, direcionam o raciocínio clínico e a intensidade das medidas preventivas ${ }^{(20,21)}$. A escala Braden Q, uma versão pediátrica da escala de Braden, foi desenvolvida por Curley e Quigley em 2004, na língua inglesa, utilizando os fatores de risco específicos para o desenvolvimento de UP em crianças ${ }^{(13)}$. Essa escala avalia os riscos por meio de dois parâmetros ${ }^{(22)}$ : o primeiro considera a intensidade e a duração da pressão por meio da avaliação da mobilidade, atividade e percepção sensorial; e o segundo, a tolerância dos tecidos pela avaliação da umidade, cisalhamento, nutrição, perfusão e oxigenação dos tecidos. Embora outras escalas tenham sido desenvolvidas a partir da observação direta de crianças, a escala de Braden Q tem sido a mais estudada e utilizada internacionalmente até o momento ${ }^{(5,22-26)}$.

Considerando-se a escassez de estudos sobre UP em crianças no Brasil e a importância para os profissionais da área de saúde do uso de um instrumento de avaliação do risco de UP na criança, planejou-se o presente estudo, cujo objetivo foi traduzir para a língua portuguesa, adaptar ao contexto cultural brasileiro e testar as propriedades de medidas, reprodutibilidade e validade da escala de Braden Q.

\section{Método}

Estudo aprovado pelo Comitê de Ética em Pesquisa da Universidade Federal de São Paulo (Unifesp). Trata-se de um estudo descritivo com tradução e adaptação (autorizadas pela autora Martha Curley) da escala para o contexto cultural brasileiro e teste de validade e reprodutibilidade, realizado na Unidade de Terapia Intensiva (UTI) Pediátrica do Hospital São Paulo. O processo de validação e adaptação transcultural da escala de Braden Q seguiu as etapas detalhadas na Figura 1.

A amostra deste estudo foi composta por 35 crianças. A fase final do processo de adaptação é o pré-teste, no qual se recomenda a aplicação da versão pré-final com sujeitos/ pacientes, de preferência entre 30 e 40 pessoas, já que o objetivo do estudo foi a tradução e a adaptação cultural da escala, com as respectivas validações de face e de conteúdo. O critério de inclusão foi estar internado na UTI Pediátrica do Hospital São Paulo, na data da pesquisa realizada pelos enfermeiros designados e ter idade entre um mês e 13 anos,

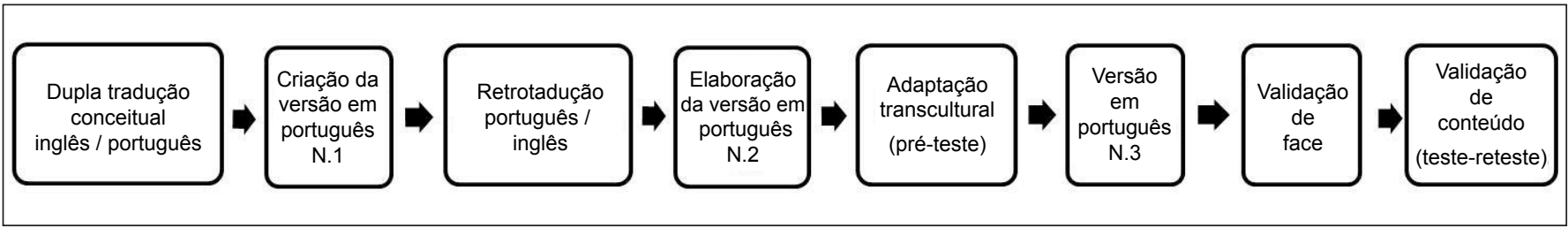

Figura 1 - Etapas do processo de validação e adaptação transcultural da escala de Braden $Q$ 
11 meses e 29 dias. O critério de exclusão foi ter idade igual ou superior a 14 anos ou menor de 1 mês de vida.

A escala de Braden $Q$ foi traduzida do original, em língua inglesa, para a língua portuguesa por dois tradutores independentes. As duas traduções foram comparadas por um grupo multidisciplinar, com quatro profissionais da saúde com nível de doutorado, criando-se a versão em português $\mathrm{n}^{\mathrm{o}} 1$. Dois tradutores independentes, fluentes na língua inglesa, não informados da existência da escala original e dos objetivos deste trabalho, realizaram a tradução reversa para a língua inglesa. O grupo multidisciplinar comparou as duas traduções reversas com a escala original na língua inglesa e criou a versão em português $\mathrm{n}^{\circ} 2$, mantendo as equivalências idiomática, semântica, conceitual e cultural. Seguiu-se a análise dos conteúdos (da tradução e da versão) e comparou-se o conteúdo da escala original, segundo o idioma e linguagem técnica empregada no Brasil, para legitimar a tradução e suas adaptações. No pré-teste, um grupo de dez enfermeiros avaliou, individualmente, a compreensão e a relevância de cada item da escala na versão em português $n^{\circ} 2$. Esses enfermeiros trabalhavam há dois anos, no mínimo, na unidade pediátrica hospitalar e foram esclarecidos sobre o estudo e requisitados a executar: a) explicação de cada item, da forma como entenderam, com suas palavras; b) sugestão de mudanças, se necessário, para torná-lo mais compreensível; c) mensuração do quanto consideravam o item relacionado ao que a escala propunha-se a medir (importância do item para avaliar o risco de desenvolver UP). Os itens foram classificados pelos enfermeiros, segundo a importância, com valores de 1 a 5 (entre nada importante a extremamente importante). A relevância foi calculada pela seguinte fórmula: $\mathrm{R}=\mathrm{FxI}(\mathrm{R}=$ relevância; $\mathrm{F}$ =frequência; $\mathrm{I}=$ importância 1 a 5). A inclusão das adaptações necessárias para a compreensão da escala, pelos enfermeiros, resultou na versão em português $n^{\circ} 3$. Esta foi aplicada em grupos consecutivos de dez enfermeiros, até a formação de um grupo composto por membros com plena compreensão de cada item da escala. A versão final, em língua portuguesa, resultou da última avaliação e do consenso do grupo multidisciplinar, que testou a validade de face (para verificar se o instrumento estava em conformidade com o projeto) e a validade de conteúdo (relevância de cada item da escala para medir o tema abordado e a importância de cada item da escala, isoladamente).

Dois enfermeiros, não participantes do pré-teste, foram selecionados e utilizaram separadamente a escala de Braden Q na avaliação, em um mesmo dia, de 35 crianças internadas na UTI do Hospital São Paulo. O primeiro enfermeiro reavaliou as crianças, após uma semana. Obtiveram-se três grupos de resultados utilizados no cálculo do índice de correlação intraclasse. O teste da reprodutibilidade da escala foi aplicado por meio de três entrevistas realizadas por esses dois enfermeiros. Para evitar a memorização dos escores, os enfermeiros foram esclarecidos quanto aos objetivos do estudo, porém não foram informados sobre a aplicação de outras entrevistas. Determinou-se, desta forma, a reprodutibilidade inter e intraobservador, para comprovar a precisão do instrumento em medir o risco para o desenvolvimento de UP em crianças.

A consistência interna (grau de correlação entre os itens da escala) foi calculada pelo coeficiente $\alpha$ de Cronbach. Para testar a validade, foi utilizado o coeficiente de correlação linear de Pearson, cujo intervalo também está compreendido entre -1 e +1 . Para testar a reprodutibilidade, foi utilizado o coeficiente de correlação intraclasses e a correlação de Spearman.

\section{Resultados}

A escala de Braden Q, traduzida e adaptada culturalmente, está descrita no Quadro 1. As médias de relevância de todos os itens permaneceram acima de 4,0 pontos na avaliação dos enfermeiros (Tabela 1). A avaliação da consistência interna dos itens da escala foi medida pelo $\alpha$ de Crombach, obtendo-se o valor de 0,936 . A Tabela 2 apresenta o valor de $\alpha$ de Crombach geral e os valores obtidos caso cada item específico fosse eliminado da escala, demonstrando o nível de correlação entre eles.

O cálculo da correlação de Spearman entre os itens da escala avaliou a reprodutibilidade por meio da aplicação da escala de Braden Q em 35 crianças da UTI Pediátrica por dois observadores, em três momentos distintos. Os resultados obtidos mostraram valores menores $(0,26-0,49)$ para o item perfusão e oxigenação tecidual quando correlacionado aos demais, indicando baixa correlação do item perfusão com os outros itens (Tabela 3).

A avaliação da reprodutibilidade intraobservador foi realizada por meio das duas avaliações de 35 pacientes pelo mesmo enfermeiro selecionado. $\mathrm{O}$ valor da correlação intraclasse obtido foi de 0,995 , apontando uma excelente reprodutibilidade. Os valores de correlação de Pearson intraobservador resultaram em $\mathrm{r}=0,9949$. $\mathrm{Na}$ avaliação da reprodutibilidade interobservadores, foram utilizadas as duas avaliações de 35 pacientes por dois enfermeiros distintos. $\mathrm{O}$ valor da correlação intraclasse obtido foi de 
0,998, evidenciando também uma excelente reprodutibilidade. $\mathrm{Na}$ análise de concordância dos valores dos escores totais, a correlação de Pearson interobservadores demonstrou $r=0,9982$. A reprodutibilidade entre as observações de um mesmo enfermeiro (intraobservador) e entre as observações de dois enfermeiros diferentes

Quadro 1 - Escala de Braden Q para avaliação do risco de úlcera por pressão em crianças

\begin{tabular}{|c|c|c|c|c|}
\hline $\begin{array}{l}\text { Mobilidade } \\
\text { Capacidade de } \\
\text { mudar e controlar a } \\
\text { posição do corpo. }\end{array}$ & $\begin{array}{l}\text { 1. Completamente } \\
\text { Imóvel: Não faz } \\
\text { mudanças, nem } \\
\text { mesmo pequenas, } \\
\text { na posição do } \\
\text { corpo ou das } \\
\text { extremidades, sem } \\
\text { ajuda. }\end{array}$ & $\begin{array}{l}\text { 2. Muito limitado: } \\
\text { Faz pequenas } \\
\text { mudanças } \\
\text { ocasionais na } \\
\text { posição do corpo } \\
\text { ou extremidades, } \\
\text { mas é incapaz de } \\
\text { fazer mudanças } \\
\text { completamente } \\
\text { sozinho. }\end{array}$ & $\begin{array}{l}\text { 3. Levemente } \\
\text { limitado: Faz } \\
\text { mudanças } \\
\text { freqüentes, embora } \\
\text { pequenas, na } \\
\text { posição do corpo ou } \\
\text { das extremidades, } \\
\text { sem ajuda. }\end{array}$ & $\begin{array}{l}\text { 4. Nenhuma } \\
\text { limitação: Faz } \\
\text { mudanças } \\
\text { importantes e } \\
\text { freqüentes na } \\
\text { posição do corpo, } \\
\text { sem ajuda. }\end{array}$ \\
\hline $\begin{array}{l}\text { ATIVIDADE } \\
\text { Grau de atividade } \\
\text { física. }\end{array}$ & $\begin{array}{l}\text { 1. Acamado: } \\
\text { Permanece no leito } \\
\text { o tempo todo. }\end{array}$ & $\begin{array}{l}\text { 2. Restrito à cadeira: } \\
\text { A capacidade de } \\
\text { deambular está } \\
\text { gravemente limitada } \\
\text { ou inexistente. Não } \\
\text { consegue sustentar } \\
\text { o próprio peso e/ } \\
\text { ou precisa de ajuda } \\
\text { para sentar-se em } \\
\text { uma cadeira ou } \\
\text { cadeira de rodas. }\end{array}$ & $\begin{array}{l}\text { 3. Deambulação } \\
\text { ocasional: Deambula } \\
\text { ocasionalmente } \\
\text { durante o dia, porém } \\
\text { por distâncias bem } \\
\text { curtas, com ou sem } \\
\text { ajuda. Passa a maior } \\
\text { parte do turno no } \\
\text { leito ou na cadeira. }\end{array}$ & $\begin{array}{l}\text { 4. Crianças } \\
\text { jovens demais } \\
\text { para deambular } \\
\text { ou deambulam } \\
\text { freqüentemente: } \\
\text { Deambula fora do } \\
\text { quarto pelo menos } \\
\text { duas vezes por dia } \\
\text { e dentro do quarto } \\
\text { pelo menos uma vez } \\
\text { a cada duas horas } \\
\text { durante as horas } \\
\text { está acordado. }\end{array}$ \\
\hline $\begin{array}{l}\text { PERCEPÇÃO } \\
\text { SENSORIAL } \\
\text { Capacidade de } \\
\text { responder de } \\
\text { maneira apropriada } \\
\text { ao desconforto } \\
\text { relacionado à } \\
\text { pressão }\end{array}$ & $\begin{array}{l}\text { 1. Completamente } \\
\text { limitada: Não } \\
\text { responde ao } \\
\text { estímulo doloroso } \\
\text { (não geme, não } \\
\text { se encolhe ou se } \\
\text { agarra), devido à } \\
\text { diminuição do nível } \\
\text { de consciência, ou } \\
\text { sedação ou limitação } \\
\text { da capacidade de } \\
\text { sentir dor na maior } \\
\text { parte da superfície } \\
\text { corporal. }\end{array}$ & $\begin{array}{l}\text { 2. Muito limitada: } \\
\text { Responde apenas } \\
\text { ao estímulo } \\
\text { doloroso. Não } \\
\text { consegue comunicar } \\
\text { desconforto, exceto } \\
\text { por gemido ou } \\
\text { inquietação; ou } \\
\text { apresenta alguma } \\
\text { disfunção sensorial } \\
\text { que limita a } \\
\text { capacidade de sentir } \\
\text { dor ou desconforto } \\
\text { em mais da metade } \\
\text { do corpo. }\end{array}$ & $\begin{array}{l}\text { 3. Levemente } \\
\text { limitada: Responde } \\
\text { aos comandos } \\
\text { verbais, mas nem } \\
\text { sempre consegue } \\
\text { comunicar o } \\
\text { desconforto ou a } \\
\text { necessidade de ser } \\
\text { mudado de posição, } \\
\text { ou apresenta alguma } \\
\text { disfunção sensorial } \\
\text { em uma ou duas } \\
\text { extremidades que } \\
\text { limita a capacidade } \\
\text { de sentir dor }\end{array}$ & $\begin{array}{l}\text { 4. Nenhuma } \\
\text { alteração: Responde } \\
\text { aos comandos } \\
\text { verbais. Não } \\
\text { apresenta déficit } \\
\text { sensorial que limite a } \\
\text { capacidade de sentir } \\
\text { ou comunicar dor ou } \\
\text { desconforto. }\end{array}$ \\
\hline $\begin{array}{l}\text { UMIDADE } \\
\text { Grau de exposição } \\
\text { da pele à umidade. }\end{array}$ & $\begin{array}{l}\text { 1. Constantemente } \\
\text { úmida: A pele fica } \\
\text { constantemente } \\
\text { úmida por suor, } \\
\text { urina, etc. A umidade } \\
\text { é percebida cada } \\
\text { vez que o paciente } \\
\text { é movimentado ou } \\
\text { mudado de posição. }\end{array}$ & $\begin{array}{l}\text { 2. Frequentemente } \\
\text { úmida: A pele está } \\
\text { frequentemente, mas } \\
\text { nem sempre, úmida. } \\
\text { A roupa de cama } \\
\text { precisa ser trocada } \\
\text { pelo menos a cada } \\
\text { oito horas. }\end{array}$ & $\begin{array}{l}\text { 3. Ocasionalmente } \\
\text { úmida: A pele está } \\
\text { ocasionalmente } \\
\text { úmida, necessitando } \\
\text { de troca de roupa } \\
\text { de cama a cada } 12 \\
\text { horas. }\end{array}$ & $\begin{array}{l}\text { 4. Raramente úmida: } \\
\text { A pele geralmente } \\
\text { está seca, as trocas } \\
\text { de fraldas são feitas } \\
\text { de rotina e as roupas } \\
\text { de cama necessitam } \\
\text { ser trocadas apenas } \\
\text { a cada } 24 \mathrm{~h}\end{array}$ \\
\hline
\end{tabular}


Quadro 1 - Continuação

\begin{tabular}{|c|c|c|c|c|}
\hline $\begin{array}{l}\text { FRICÇÃO E } \\
\text { CISALHAMENTO } \\
\text { Fricção: a pele } \\
\text { se move contra } \\
\text { as estruturas de } \\
\text { suporte. } \\
\text { Cisalhamento: a pele } \\
\text { e a superfície óssea } \\
\text { adjacente deslizam } \\
\text { uma sobre a outra. }\end{array}$ & $\begin{array}{l}\text { 1. Problema } \\
\text { importante: A } \\
\text { espasticidade, a } \\
\text { contratura, o prurido } \\
\text { ou a agitação levam } \\
\text { a criança debater-se } \\
\text { no leito e há fricção } \\
\text { quase constante. }\end{array}$ & $\begin{array}{l}\text { 2. Problema: } \\
\text { Necessita de } \\
\text { ajuda moderada } \\
\text { a máxima para se } \\
\text { mover. É impossível } \\
\text { se levantar } \\
\text { completamente } \\
\text { sem deslizar sobre } \\
\text { os lençóis do } \\
\text { leito ou cadeira, } \\
\text { necessitando de } \\
\text { reposicionamento } \\
\text { frequente com } \\
\text { o máximo de } \\
\text { assistência. }\end{array}$ & $\begin{array}{l}\text { 3. Problema } \\
\text { Potencial: } \\
\text { Movimenta-se } \\
\text { com dificuldade ou } \\
\text { necessita de mínima } \\
\text { assistência. Durante } \\
\text { o movimento, } \\
\text { provavelmente } \\
\text { ocorre atrito entre } \\
\text { a pele e os lençois, } \\
\text { cadeira, coxins ou } \\
\text { outros dispositivos. } \\
\text { A maior parte do } \\
\text { tempo mantém } \\
\text { uma posição } \\
\text { relativamente boa } \\
\text { na cadeira e no leito, } \\
\text { mas ocasionalmente } \\
\text { escorrega. }\end{array}$ & $\begin{array}{l}\text { 4. Nenhum problema } \\
\text { aparente: Capaz } \\
\text { de levantar-se } \\
\text { completamente } \\
\text { durante uma } \\
\text { mudança de } \\
\text { posição. Movimenta- } \\
\text { se sozinho na } \\
\text { cadeira e no } \\
\text { leito, e tem força } \\
\text { muscular suficiente } \\
\text { para levantar-se } \\
\text { completamente } \\
\text { durante o } \\
\text { movimento. Mantém } \\
\text { uma posição } \\
\text { adequada no leito e } \\
\text { na cadeira o tempo } \\
\text { todo. }\end{array}$ \\
\hline $\begin{array}{l}\text { NUTRIÇÃO } \\
\text { Padrão habitual de } \\
\text { consumo alimentar. }\end{array}$ & $\begin{array}{l}\text { 1. Muito pobre: Em } \\
\text { jejum e/ou mantido } \\
\text { com ingesta hídrica } \\
\text { ou hidratação IV por } \\
\text { mais de } 5 \text { dias ou } \\
\text { albumina < } 2,5 \mathrm{mg} / \mathrm{dl} \\
\text { ou nunca come uma } \\
\text { refeição completa. } \\
\text { Raramente come } \\
\text { mais da metade } \\
\text { de algum alimento } \\
\text { oferecido. O } \\
\text { consumo de } \\
\text { proteínas inclui } \\
\text { apenas duas } \\
\text { porções de carne ou } \\
\text { derivados de leite } \\
\text { por dia. Ingere pouco } \\
\text { líquido. Não ingere } \\
\text { suplemento dietético } \\
\text { líquido. }\end{array}$ & $\begin{array}{l}\text { 2. Inadequada: Dieta } \\
\text { líquida por sonda } \\
\text { ou NPP que fornece } \\
\text { calorias e minerais } \\
\text { insuficientes } \\
\text { para a idade ou } \\
\text { albumina < } 3 \mathrm{mg} / \\
\text { dl ou raramente } \\
\text { come uma a } \\
\text { refeição completa. } \\
\text { Geralmente } \\
\text { come apenas a } \\
\text { metade de algum } \\
\text { alimento oferecido. } \\
\text { O consumo de } \\
\text { proteínas inclui } \\
\text { apenas três } \\
\text { porções de carne } \\
\text { ou derivados } \\
\text { de leite por dia. } \\
\text { Ocasionalmente } \\
\text { ingere suplemento } \\
\text { dietético. }\end{array}$ & $\begin{array}{l}\text { 3. Adequada: } \\
\text { Dieta por sonda ou } \\
\text { NPP que fornece } \\
\text { calorias e minerais } \\
\text { suficientes para } \\
\text { a idade ou come } \\
\text { mais da metade } \\
\text { da maioria das } \\
\text { refeições. Consome } \\
\text { um total de quatro } \\
\text { porções de proteínas } \\
\text { (carne, derivados } \\
\text { de leite) por dia. } \\
\text { Ocasionalmente } \\
\text { recusa uma refeição, } \\
\text { mas geralmente } \\
\text { toma suplemento } \\
\text { dietético, se } \\
\text { oferecido. }\end{array}$ & $\begin{array}{l}\text { 4. Excelente: Dieta } \\
\text { geral que fornece } \\
\text { calorias suficientes } \\
\text { para a idade. Por } \\
\text { exemplo, come/ } \\
\text { bebe a maior parte } \\
\text { de cada refeição/ } \\
\text { alimentação. } \\
\text { Nunca recusa uma } \\
\text { refeição. Geralmente } \\
\text { come um total de } \\
\text { quatro ou mais } \\
\text { porções de carne e } \\
\text { derivados de leite. } \\
\text { Ocasionalmente, } \\
\text { come entre as } \\
\text { refeições. Não } \\
\text { necessita de } \\
\text { suplementação. }\end{array}$ \\
\hline $\begin{array}{l}\text { PERFUSÃO } \\
\text { TECIDUAL E } \\
\text { OXIGENAÇÃO }\end{array}$ & $\begin{array}{l}\text { 1. Extremamente } \\
\text { comprometida: } \\
\text { Hipotenso (PAM <50 } \\
\text { mmHg; <40 mmHg } \\
\text { em recém-nascido) } \\
\text { ou o paciente não } \\
\text { tolera as mudanças } \\
\text { de posição. }\end{array}$ & $\begin{array}{l}\text { 2. Comprometida: } \\
\text { Normotenso. } \\
\text { Apresenta saturação } \\
\text { de oxigênio <95\% ou } \\
\text { a hemoglobina <10 } \\
\text { mg/dl ou o tempo de } \\
\text { enchimento capilar } \\
>2 \text { segundos. } \mathrm{O} \text { pH } \\
\text { sérico }<7,40 \text {. }\end{array}$ & $\begin{array}{l}\text { 3. Adequada: } \\
\text { Normotenso. } \\
\text { Apresenta saturação } \\
\text { de oxigênio < } 95 \% \text { ou } \\
\text { a hemoglobina < } 10 \\
\text { mg/dl ou o tempo de } \\
\text { enchimento capilar } \\
>2 \text { segundos. O pH } \\
\text { sérico é normal. }\end{array}$ & $\begin{array}{l}\text { 4. Excelente: } \\
\text { Normotenso. } \\
\text { Apresenta saturação } \\
\text { de oxigênio }>95 \% \text {, } \\
\text { a hemoglobina } \\
\text { normal e o tempo de } \\
\text { enchimento capilar } \\
<2 \text { segundos. }\end{array}$ \\
\hline
\end{tabular}

Variação - 7-28 pontos. Escore 28: sem risco de úlcera de pressão; Escore 7: risco máximo. 
(interobservadores) foi demonstrada. Portanto, o instrumento foi reprodutível.

$\mathrm{Na}$ validade de face, por consenso, a equipe multidisciplinar estabeleceu que o instrumento mensura o risco de uma criança desenvolver UP. Na validade de conteúdo, por consenso, a equipe multidisciplinar julgou o conteúdo das questões do instrumento, concluindo que, realmente, mensura os riscos de desenvolvimento de UP na criança.

Tabela 1 - Índice de relevância de cada item pontuado por cada enfermeiro

\begin{tabular}{lccc}
\hline & Média & Mínimo & Máximo \\
\hline Mobilidade & 4,83 & 3 & 5 \\
Atividade & 4,43 & 3 & 5 \\
Percepção sensorial & 4,63 & 3 & 5 \\
Umidade & 4,60 & 3 & 5 \\
Fricção e cisalhamento & 4,80 & 3 & 5 \\
Nutrição & 4,20 & 3 & 5 \\
Perfusão e oxigenação & 4,50 & 3 & 5 \\
\hline
\end{tabular}

Tabela 2 - Valores de $\alpha$ de Crombach para cada item da escala

\begin{tabular}{lc}
\hline Item específico eliminado & $\begin{array}{c}\text { Valor do } \alpha \text { de } \\
\text { Crombach* }^{*}\end{array}$ \\
\hline Mobilidade & 0,918 \\
Atividade & 0,927 \\
Percepção sensorial & 0,916 \\
Umidade & 0,923 \\
Fricção e cisalhamento & 0,913 \\
Nutrição & 0,926 \\
Perfusão e oxigenação & 0,953 \\
Total** & 0,936 \\
\hline
\end{tabular}

*Valor obtido caso o item correspondente fosse eliminado da escala;

**Valor obtido sem a retirada de nenhum dos itens da escala.

\section{Discussão}

Entre os diversos estudos epidemiológicos da ocorrência de UP $\mathrm{P}^{(2,15-19)}$, Baldwin obteve taxas de 0,29\% de incidência em uma investigação nacional nos Estados Unidos em crianças hospitalizadas ou não ${ }^{(2)}$. Neidig et al encontraram incidência de 4,8\% de UP em crianças com até 36 meses de idade, em pós-operatório de cirurgia cardíaca ${ }^{(17)}$ e Curley e Quigley descreveram essa intercorrência em 27\% de crianças internadas em unidades de terapia intensiva pediátricas $^{(13)}$.

As UP são associadas ao aumento da permanência do paciente no hospital. A avaliação de risco é um indicador importante para predizer a formação da úlcera e deve ser realizada na admissão do paciente, como base para a sua profilaxia ${ }^{(21,24)}$. A escolha de um instrumento apropriado para avaliar e identificar os pacientes em risco é uma etapa importante para determinar as medidas preventivas corretas e o tratamento de UP ${ }^{(20-22)}$. As equipes de profissionais beneficiam-se do uso da escala de avaliação do risco, como um guia. Além da uniformidade nos resultados da avaliação, mantendo o risco sob foco, esta possibilita padronizar condutas e otimizar a utilização de recursos materiais e humanos na prevenção da UP na criança.

Existem várias escalas para avaliar o risco de pacientes adultos desenvolverem UP, dentre as quais a escala de Braden $^{(21,27)}$. Curley et a ${ }^{(14)}$ adaptaram a escala de Braden para a população pediátrica, surgindo, assim, a escala de Braden Q, que emprega fatores de risco específicos para a Pediatria $^{(22,27)}$.

A escala de Braden Q avalia a perfusão e a oxigenação tecidual, que englobam a saturação de oxigênio, o nível de hemoglobina e o $\mathrm{pH}$ sérico ${ }^{(13)}$. Os parâmetros referentes à intensidade e à duração da pressão incluem os itens mobilidade, atividade e percepção sensorial. Cada um dos sete itens

Tabela 3 - Correlação de Spearman entre os itens da escala de Braden $Q$

\begin{tabular}{lccccccc}
\hline & Mobilidade & Atividade & $\begin{array}{c}\text { Percepção } \\
\text { sensorial }\end{array}$ & Umidade & $\begin{array}{c}\text { Fricção e } \\
\text { cisalhamento }\end{array}$ & Nutrição & $\begin{array}{c}\text { Perfusão e } \\
\text { oxigenação }\end{array}$ \\
\hline Mobilidade & 1,00 & 0,81 & 0,81 & 0,80 & 0,88 & 0,76 & 0,47 \\
Atividade & - & 1,00 & 0,78 & 0,80 & 0,79 & 0,72 & 0,25 \\
Percepção sensorial & - & - & 1,00 & 0,84 & 0,88 & 0,79 & 0,40 \\
Umidade & - & - & - & 1,00 & 0,84 & 0,74 & 0,26 \\
Fricção e cisalhamento & - & - & - & - & 1,00 & 0,77 & 0,45 \\
Nutrição & - & - & - & - & - & 1,00 & 0,49 \\
Perfusão e oxigenação & - & - & - & - & - & & 1,00 \\
\hline
\end{tabular}


que a compõem são pontuados de 1 a 4 , e o escore total varia de 7 (maior risco) a 28 (ausência de risco) $)^{(14)}$.

$\mathrm{O}$ primeiro item da escala é a mobilidade, que gradua a capacidade de mudar e controlar a posição do corpo. A mobilidade contribui para o bem-estar físico e psíquico de todo indivíduo, pois o paciente imóvel não alivia a pressão nas regiões de proeminências ósseas ${ }^{(14)}$. $\mathrm{O}$ item atividade avalia o grau de ação ou trabalho físico da criança, ou seja, se permanece acamada ou alterna período sentada em cadeira, ou se tem ou não idade suficiente para deambular ${ }^{(14)}$. A percepção sensorial é um item que pontua a forma como a criança responde ao estímulo doloroso e ao desconforto relacionado com a pressão, variando desde a incapacidade de resposta (no caso de sedação ou alguma disfunção sensorial que limite a capacidade de sentir e expressar a dor) até a percepção sensorial normal ${ }^{(14)}$. A diminuição do nível de consciência está associada, direta ou indiretamente, à percepção sensorial, à mobilidade e à atividade. $\mathrm{O}$ comprometimento da percepção do desconforto causado pela pressão, motivado pelo estado de alerta para a movimentação espontânea, pela restrição física por motivos de segurança ou pelo uso de sedativos, impede o alívio da pressão sobre os tecidos nas áreas de proeminências ósseas, com significante aumento do risco de desenvolvimento de $\mathrm{UP}^{(14)}$.

Na escala de Braden Q, a tolerância da pele e de estruturas de suporte abrange os itens: umidade, fricção e cisalhamento, nutrição e perfusão tissular e oxigenação ${ }^{(14)}$. A avaliação da umidade pontua a exposição da pele à transpiração, à urina e outras líquidos que permaneçam em contato indevido com a pele. É medida pela quantidade de vezes em que são trocados os lençóis e as fraldas ${ }^{(14)}$. A maceração e a lesão, causadas pela umidade excessiva, destroem a barreira natural da epiderme. As incontinências urinária e anal são fatores potenciais de risco para $\mathrm{UP}^{(2,28)}$. Em uma ampla revisão da literatura, também se observou que o risco da lesão é 22 vezes maior em pacientes com incontinência anal e urinária, comparados a pacientes continentes ${ }^{(29,30)}$. Cerca de $30 \%$ das crianças normais de quatro anos e $10 \%$ das crianças de seis anos não atingem a continência noturna ${ }^{(30)}$. Crianças também são muito suscetíveis à presença de lesões em dobras do corpo (como ocorre por excesso de tecido adiposo), devido ao acúmulo de suor ou de outros líquidos que permaneçam em contato com a pele. A maceração causada pela umidade pode combinarse à pressão, principalmente na presença de tiras, cordões, coletores ou suportes no corpo. A fricção ocorre em pacientes incapazes de reposicionar-se sem ajuda no leito, ao passo que o cisalhamento é causado pela interação da gravidade e da fricção, que exercem forças paralelas na pele. Enquanto a gravidade empurra o corpo para baixo, a resistência do paciente sobre a superfície da cama ou cadeira (fricção) impede a declinação do corpo, e a pele move-se contra as estruturas de suporte, principalmente em proeminências ósseas. Esse item contempla a agitação da criança, a eventual existência de prurido, a maneira como se movimenta, considerando-se o atrito com a pele ${ }^{(14)}$. Muitas crianças que utilizam cadeira de rodas exercem atividades como brincadeiras e recreação, incentivadas, mesmo no ambiente hospitalar, por trazerem bem-estar. Tais atividades, contudo, somando-se às deficiências motoras e sensoriais dos pacientes, podem levar a maior cisalhamento na região isquiática e sacral. A nutrição é classificada de acordo com o número de refeições e com a via de administração da dieta. Nesse item, considera-se o nível de albumina e a necessidade calórica por idade, se a criança está ganhando peso e se há necessidade de adequação da dieta. A má nutrição é um dos fatores primários para o desenvolvimento de UP, por contribuir para diminuir a tolerância do tecido à pressão ${ }^{(30)}$. As necessidades calóricas na infância são duas a três vezes maiores do que no adulto. No primeiro ano de vida, $40 \%$ dessas calorias são empregadas nas demandas do processo de crescimento e de desenvolvimento ${ }^{(7)}$. A perfusão e a oxigenação quantificam a perfusão dos tecidos de acordo com a saturação de oxigênio, com o nível de hemoglobina e o pH sérico. A perfusão e a oxigenação tecidual são aspectos intrínsecos da tolerância da pele; a baixa pressão arterial pode predispor os pacientes a desenvolver UP, quando comparados aos normotensos. Esse é um item importante na escala para crianças com quadro clínico grave, em uso de vasopressores ${ }^{(30)}$. O valor da hemoglobina aumenta com a idade, mas mantém-se praticamente constante durante a faixa de idade em que a anemia por deficiência de ferro é mais comum. Entre seis meses e cinco anos de idade, o limite inferior da normalidade para o valor da hemoglobina é de $11 \mathrm{~g} / \mathrm{dL}^{(28)}$. O pH sérico em criança é considerado normal nos valores de 7,35 a 7,45. Quando ocorre o aumento do teor de bicarbonato sanguíneo ou a diminuição da concentração de gás carbônico, o pH torna-se maior que o normal, indicando alcalose. A diminuição da concentração de bicarbonato do sangue ou o aumento da concentração de gás carbônico causa redução do $\mathrm{pH}$, indicando, assim, a acidose. $\mathrm{Na}$ ausência desses dados, utiliza-se a saturação de oxigênio como parâmetro de avaliação ${ }^{(14)}$.

$\mathrm{O}$ alto índice de relevância na escala de Braden $\mathrm{Q}$ demonstrou o bom entendimento dos enfermeiros na fase de adaptação cultural. A avaliação da reprodutibilidade da escala 
de Braden Q é importante, pois será utilizada por enfermeiros - classe profissional que realizou esta etapa do estudo.

A análise dos escores obtidos com a aplicação da escala evidenciou altos índices de correlação entre as observações do mesmo avaliador. O coeficiente de correlação intraclasse mostrou valores semelhantes entre o mesmo observador em períodos diversos e entre diferentes observadores, respectivamente, 0,995 e 0,998 , não havendo diferenças significantes. A reprodutibilidade indica ser viável a aplicação desse instrumento, adaptado à realidade brasileira, nas diversas unidades pediátricas do país, independentemente dos turnos de enfermagem.

Quanto à consistência interna, esta é verificada pela avaliação de correlação entre os itens de uma escala, sendo calculado estatisticamente por meio do coeficiente $\alpha$ de Cronbach. Se os itens de uma escala apresentam alto nível de correlação, assume-se que medem um único conceito e, portanto, apresentam consistência interna. A consistência interna dos itens da escala de Braden Q na sua versão final para a língua portuguesa mostraram índice $\alpha$ de Crombach de 0,936 , indicando consistência interna do instrumento.

Em relação à reprodutibilidade intra e interobservadores, o instrumento estatístico empregado foi a correlação intraclasse, medida pela correlação de Spearman entre os itens da escala, cujo resultado varia entre -1 e +1 . Quanto mais próximo de +1 , melhor a reprodutibilidade intra ou interobservador. Assim como na análise da consistência interna pelo $\alpha$ de Crombach, o teste de correlação de Spearman obteve, para o item perfusão tecidual e oxigenação, valores menores, indicando baixa correlação deste com os demais (Tabela 3). Porém, tais resultados não comprometem a escala, que manteve, como um todo, seu valor significante de avaliar o risco de UP em crianças.

Estudo de coorte feito por Braden et al ${ }^{(14)} \mathrm{com} 322$ crianças entre 21 dias e 8 anos, internadas em unidades de terapia intensiva pediátricas norte-americanas (excluídas crianças com cardiopatias congênitas), indicou a pontuação (escore) 16 como alto risco para o desenvolvimento de UP. Para esse escore, a versão em português da escala demonstrou sensibilidade de 0,88 e especificidade de 0,58.

Testes adicionais das propriedades psicométricas são altamente recomendaveis, com amostras maiores e homogêneas. A escala de Braden $\mathrm{Q}$ traduzida é direcionada a pacientes em estado crítico e não foi elaborada para neonatos e préadolescentes ou adolescentes, o que limita a sua utilização à faixa etária compreendida entre um mês de vida e escolares. Como as características de populações internadas podem variar em suas condições clínicas, também a leitura por profissionais de diferentes locais ou unidades deve ser avaliada, sendo necessários treinamentos da equipe e consenso para possibilitar o seu uso. Entende-se, portanto, que deva ainda ser aplicada a populações diversas na Pediatria, para estabelecer o alcance de sua capacidade em prever o risco de desenvolvimento de UP. Finalmente, vale considerar que os itens da escala podem ser analisados individualmente, quando há comprometimento, para otimizar e facilitar as intervenções necessárias.

Assim, conclui-se que a escala de Braden Q, traduzida e adaptada à cultura brasileira, demonstrou credibilidade (validade de face), além de ser um instrumento válido, reprodutível e confiável para avaliar o risco do desenvolvimento de UP em crianças, constituindo-se em ferramenta útil para nortear as ações condizentes com o risco de cada paciente, individualizando a assistência. A validação inicial pressupõe a continuidade de pesquisas com amostras maiores para a devida comparação dos resultados na realidade brasileira. Os escores devem ser estabelecidos e validados de acordo com o risco para populações com diferentes características clínicas e demográficas na Pediatria, ou seja, doenças específicas, comprometimento físico e mental, faixa etária e atividades, entre outros.

\section{Referências bibliográficas}

1. Zollo MB, Gostisha ML, Berens RJ, Schmidt JE, Weigle CG. Altered skin integrity in children admitted to a pediatric intensive care unit. J Nurs Care Qual 1996;11:62-7.

2. Baldwin KM. Incidence and prevalence of pressure ulcers in children. Adv Skin Wound Care 2002;15:121-4.

3. Donabedian A. The role of outcomes in quality assessment and assurance. QRB Qual Rev Bull 1992;18:356-60.

4. Meraviglia M, Becker H, Grobe SJ, King M. Maintence of skin integrity as a clinical indicator of nursing care. Adv Skin Wound Care 2002;15:24-9.

5. Butler CT. Pediatric skin care: guidelines for assessment, prevention, and treatment. Pediatr Nurs 2006;32:443-50.
6. Waterlow JA. Pressure sore risk assessment in children. Paediatr Nurs 1997;9:21-4.

7. Ferreira LM, Calil JA. Etiopatologia e tratamento das úlceras por pressão. Diagn Tratamento 2001;6:36-40.

8. National Pressure Ulcer Advisory Panel [homepage on the internet]. Pressure Ulcer Stages Revised by NPUAP [cited 2009 Oct 12]. Available from: http:// www.npuap.org/pr2.htm

9. Gershan LA, Esterly NB. Scarring alopecia in neonates as a consequence of hypoxaemia- hypoperfusion. Arch Dis Child 1993;68:591-3.

10. Girouard K, Harrisson MB, VanDenKerkof E. The symptom of pain with pressure ulcers: a review of the literature. Ostomy Wound Manage 2008;54:30-40. 
11. Bernal Bellón AC, Nieto Torres MC. Úlceras por presión en niños evaluación del riesgo em la Unidad de Cuidado Intensivo Pediátrico. Aquichan 2004:4:10-7.

12. Quigley SM, Curley MA. Skin integrity in the pediatric population: preventing and managing pressure ulcers. J Soc Pediatr Nurs 1996;1:7-18.

13. Curley MA, Quigley SM, Lin M. Pressure ulcers in pediatric intensive care: incidence and associated factors. Pediatr Crit Care Med 2003;4:284-90.

14. Curley MA, Razmus IS, Roberts KE, Wypij D. Predicting pressure ulcer risk in pediatric patients: the Braden Q Scale. Nurs Res 2003;52:22-33.

15. Schindler CA, Mikhailov TA, Fischer K, Lukasiewicz G, Kuhn EM, Duncan L. Skin integrity in critically ill and injured children. Am J Crit Care 2007;16: 568-74.

16. Samaniego IA. A sore spot in pediatrics: risk factors for pressure ulcers. Derm Nurs 2004;16:153-9.

17. Neidig JR, Kleiber C, Oppliger RA. Risk factors associated with pressure ulcers in the pediatric patient following open--heart surgery. Prog Cardiovasc Nurs 1989;4:99-106.

18. Dixon M, Ratliff C. Pediatric pressure ulcer prevalence--one hospital's experience. Ostomy Wound Manage 2005;51:48-50.

19. McLane KM, Bookout K, McCord S, McCain J, Jefferson LS. The 2003 national pediatric pressure ulcer and skin breakdown prevalence survey: a multisite study. J Wound Ostomy Continence Nurs 2004;31:168-78.

20. Pancorbo-Hidalgo PL, Garcia-Fernandez FP, Lopez-Medina IM, Alvarez-Nieto C. Risk assessment scales for pressure ulcer prevention: a systematic review. J Adv Nurs 2006;54:94-110.
21. Ayello EA, Braden B. Clinical management extra: pressure ulcers. How and why to do pressure ulcer risk assessment. Advances in Skin \& Wound Care 2002;15:125-31.

22. Gray M. Which pressure ulcer risk scales are valid and reliable in a pediatric population? J Wound Ostomy Continence Nurs 2004;31:157-60.

23. Beaton DE, Bombardier C, Guillemin F, Ferraz MB. Guidelines for the process of cross-cultural adaptation of self-report measures. Spine (Phila Pa 1976) 2000;25:3186-91.

24. Pasek TA, Geyser A, Sidoni M, Harris P, Warner JA, Spence A et al. Skin care team in the pediatric intensive care unit: a model for excellence. Crit Care Nurse 2008;28:125-35.

25. Noonan C, Quigley C, Curley MA. Skin integrity in hospitalized infants and children: a prevalence survey. J Pediatr Nurs 2006;21:445-53.

26. Schlüer $A B$, Cignacco $E$. Escarres chez les enfants - un problème à prendre au sérieux. Krankenpflege Soins Infirmiers 2008;101:38-41.

27. Willock J, Anthony D, Richardson J. Inter-rater reliability of Glamorgan Paediatric Pressure Ulcer Risk Assessment Scale. Paediatr Nurs 2008;20:14-9.

28. Blanes L, Duarte IS, Calil JA, Ferreira LM. Avaliação clínica e epidemiológica das úlceras por pressão em pacientes internados no Hospital São Paulo. Rev Assoc Med Bras 2004;50:182-7.

29. Rodriguez-Key M, Alonzi A. Nutrition, skin integrity, and pressure ulcer healing in chronically ill children: an overview. Ostomy Wound Manage 2007;53:56-66.

30. Braden B, Bergstrom N. A conceptual schema for the study of the etiology of pressure sores. Rehabil Nurs 1987;12:8-12. 